Research Paper

\title{
Construction and Identification of Recombinant HEK293T Cell Lines Expressing Non-structural Protein 1 of Zika Virus
}

Jun Liu', 2*, Pengfei Wan ${ }^{1 *}$, Qingqing $\mathrm{Li}^{1 *}$, Xiaoxin $\mathrm{Li}^{1}$, Andrew $\mathrm{Li}^{3}$, Huangyao Chen ${ }^{1}$, Jingjing Li1 ${ }^{1}$, Wenhan Liang ${ }^{1}$, Haifa Zheng ${ }^{4 凶}$, Weiwang Gu ${ }^{2}$, Hongwei Li $^{1}{ }^{\bowtie}$

1. School of Laboratory Medicine and Biotechnology, Southern Medical University, Guangzhou, Guangdong, China;

2. Institute of Comparative Medicine and Center of Laboratory Animals, Southern Medical University, Guangzhou, Guangdong, China;

3. Department of Biomedical Engineering, The Johns Hopkins University School of Medicine, Baltimore, USA;

4. Beijing Minhai Biotechnology CO. LTD, Beijing, China.

* These authors contributed equally to this work.

$\triangle$ Corresponding authors: Haifa Zheng, Ph.D., Beijing Minhai Biotechnology CO.LTD, No.1 Simiao Road, Biotechnology and Pharmaceuticals Industrial Base, Daxing District, Beijing 102600, China Phone: 86-10-59613588; E-mail: zhenghaifa@sina.com Weiwang Gu, MS, Institute of Comparative Medicine and Center of Laboratory Animals, Southern Medical University, 1023 South Shatai Road, Guangzhou, Guangdong 510515, China. Phone: 86-20-61648043; E-mail: guww100@163.com Hongwei Li, Ph.D., School of Laboratory Medicine and Biotechnology, Southern Medical University, 1023 South Shatai Road, Guangzhou, Guangdong 510515, China. Phone: 86-20-61648555; Fax: 86-20-61648555; E-mail: lihw@smu.edu.cn

(c) Ivyspring International Publisher. This is an open access article distributed under the terms of the Creative Commons Attribution (CC BY-NC) license (https://creativecommons.org/licenses/by-nc/4.0/). See http://ivyspring.com/terms for full terms and conditions.

Received: 2017.04.05; Accepted: 2017.07.05; Published: 2017.09.04

\begin{abstract}
Background: Zika virus (ZIKV) infection has become a major public health problem all around the world. Early diagnosis of Zika infection is important for better management of the disease. Non-structural protein 1 (NSI) is a potential biomarker for ZIKV infections. The purpose of this study was to produce the ZIKV NSI protein for establishing serological diagnostic methods for ZIKV.

Methods: The cDNA fragment encoding a chimeric protein composed of murine IgK signal peptide, NSI and histidine tag was synthesized and cloned into the lentiviral expression vector pLV-eGFP. The resulting expression vector PLV-eGFP-ZIKV-NSI was packaged and transduced into human embryonic kidney (HEK) 293T cells and clonal cell lines with NS1 gene were generated from the tranduced cells by limiting dilution. Over expressed recombination NSI ( $\mathrm{NNS1}$ ) fusion protein was purified by nickel affinity chromatography. Mice immunization and enzyme-linked immunosorbent assay (ELISA) were carried out to evaluate the immunogenicity of rNS1.

Results: Western blot analysis revealed that the reconstituted cells stably expressed and secreted high levels of approximately 45-kDa NSI, and no significant changes were observed in green fluorescent protein (GFP) fluorescence ratio and fluorescence intensity. The scanned gels showed that the purity of the purified $\mathrm{rNS1}$ was $99.42 \%$. BALB/c mice were then immunized with purified $\mathrm{rNSI}$ and a high level of antibodies against NSI was elicited in the mice.

Conclusion: Overall, recombinant NSI proteins were successfully purified and their antigenicity was assessed. Immunization of mice with recombinant proteins demonstrated the immunogenicity of the NSI protein. Thus, the generated recombinant NSI can be potentially used in the development of serological diagnostic methods for ZIKV.
\end{abstract}

Key words: ZIKV, Non-structural protein 1, HEK293T cell, Secretory expression, Purification.

\section{Background}

Zika virus (ZIKV) infection has become a major public health problem in the tropical and sub-tropical regions. It has been presented as an international public health emergency by the World Health
Organization [1]. A comprehensive study conducted by França has demonstrated that there is a high correlation between ZIKV infection and neurodevelopmental disorders in newborn babies [2]. 
The incidence of microcephaly in newborns increased sharply in the ZIKV epidemic area, especially in Brazil [3]. Additionally, a great number of Guillain-Barré syndrome patients was reported in Colombia, where ZIKV highly prevalent, between November, 2015 and March, 2016 [4]. Epidemiological data showed that in 401 cases of ZIKV infected patients with secondary nervous system disease, $67 \%$ patients were diagnosed with Guillain-Barré syndrome [5].

The ZIKV, mainly transmitted by Aedes aegypti mosquitos, is a member of the Flaviviridae family. The ZIKV genome consists of single strand RNA (+ssRNA) which encodes three structural (C, prM/M, E) and seven non-structural proteins (NS1, NS2A, NS2B, NS3, NS4A, NS4B, NS5). Currently, there is no cure and preventive vaccines are still under clinical trials [6]. Early diagnosis is critical in preventing ZIKV transmission and can save valuable time for patients when receiving treatment for symptoms. Among the flaviviruses diagnostic methods, NS1 protein or antibody detection is the primary choice [7-9]. During acute infection phase, the virus expresses the NS1 protein, which gets released into blood. Libraty and colleagues demonstrated that high level of NS1 antigenemia has also been associated with more severe clinical presentations in dengue infected patients [10]. NS1 is a highly conserved non-structural protein among flaviviruses, and is therefore a main target for differential diagnostic tests. However, there are no NS1 antigen/antibody enzyme-linked immunosorbent assay (ELISA) kits commercially available for the early diagnosis of ZIKV.

Developing a functional protein for ZIKV diagnosis is not trivial. Post-translational modifications play crucial roles in preserving biological functions of proteins. The recombinant protein produced in bacterial expression systems is usually not soluble and loses structural and immunological features of the native viral protein [11]. Expression systems utilizing mammalian cells for recombinant proteins are able to introduce proper protein folding, post-translational modifications, and product assembly, which are important for complete biological activity. In this study, a lentiviral vector was utilized to achieve stable and secretory expression of ZIKV NS1 in HEK 293T cells.

\section{Methods}

\section{Cells and plasmids}

Human embryonic kidney (HEK) 293T cells (ATCC, Manassas, VA) were cultured in Dulbecco's modified Eagle's medium (DMEM) (Hyclone, Logan, UT) supplemented with $10 \%$ fetal bovine serum (FBS) (Hyclone), 100 units $/ \mathrm{ml}$ penicillin, $100 \mathrm{mg} / \mathrm{ml}$ streptomycin (Invitrogen, USA) and maintain in 5\% carbon dioxide $\left(\mathrm{CO}_{2}\right)$ at $37^{\circ} \mathrm{C}$.

pLV-eGFP containing Cytomegalovirus (CMV) promoter was constructed in this laboratory. The packaging plasmids pMD2.G and psPAX2 were obtained from Dr. Junming Yue (Department of Pathology, University of Tennessee Health Science Center). The cDNA fragment of the ZIKV NS1 (Haiti strain, GenBank: KU509998.3) was obtained by chemical synthesis (IGE Biotechnology, CHN), and cloned into the lentiviral expression plasmid pLV-eGFP. The resulting plasmid was designated as pLV-eGFP-Zika-NS1 (Figure 1), which was identified by restriction enzyme digestion and partial sequencing.

\section{Generation of lentiviral vectors}

The lentiviral vectors were packaged by adopting the three-plasmid packaging system [12]. On day one, a total of $6 \times 10^{6} 293 \mathrm{~T}$ cells were seeded in a $100 \mathrm{~mm}$ dish. On day 2, a transfection mix was made as the following: a solution of $500 \mu \mathrm{L}$ was first prepared consisting of $1.25 \mu \mathrm{g}$ of shuttle plasmid pMD2.G, $3.75 \mu \mathrm{g}$ of packaging plasmid psPAX2, $5 \mu \mathrm{g}$ of transfer expression plasmid pLV-eGFP-ZIKV-NS1 or pLV-eGFP, and $125 \mu \mathrm{l}$ of $2 \mathrm{mM}$ calcium chloride $\left(\mathrm{CaCl}_{2}\right)$ in deionized distilled water; $\mathrm{CaCl}_{2} / \mathrm{DNA}$ was then added dropwise while vortexing to equal volume of $2 \times$ Hepes (HBS) for a total of $1 \mathrm{~mL}$. This mix was added to the dish, and the cells maintained in 5\% $\mathrm{CO}_{2}$ at $37^{\circ} \mathrm{C}$. The GFP expression was observed by fluorescent microscopy after $48 \mathrm{~h}$, and the packaged recombinant lentiviruses (LV-eGFP-ZIKV-NS1 and LV-eGFP) were harvested from the supernatant of cell cultures at $48 \mathrm{~h}$ post transfection. The lentivirus titer was detected using real-time PCR as described previously [13].

\section{Establishment of Recombinant Cell Lines}

A total of $4 \times 10^{4} 293 \mathrm{~T}$ cells / well were prepared in a 24-wells plate. On the following day, the cells in each well were transduced with packaged recombinant lentivirus at a multiplicity of infection (MOI) of 10 (10 viral genomes per cell) in DMEM medium containing 10\% FBS with $6-8 \mu \mathrm{g} / \mathrm{ml}$ hexadimethrine bromide (Polybrene, Sigma, Germany). After $24 \mathrm{~h}$, transduction media was replaced with fresh DMEM with 10\% FBS and incubated for $3-5$ days at $37^{\circ} \mathrm{C}$ and $5 \% \quad \mathrm{CO}_{2}$. The transduced cells were then clonally expanded by limiting dilution. The cells were plated in three plates at a density of 0.8 cell/well in 100 $\mu$ l of DMEM containing $10 \%$ FBS. Two to three weeks later, clones in good condition were picked and cultured. The cells were passaged once every three days at a ratio of 1:10. 
Gene expression of the transduced cells was evaluated by western blot using an anti-NS1 monoclonal antibody (Zoonogen, $\mathrm{CHN}$ ).

\section{Purification of rNS1}

For rNS1 purification, HEK-293T-ZIKV-NS1-7 cells culture supernatant was harvested and filtered using $0.45 \mu \mathrm{m}$ syringe filters, and then loaded on the nickel affinity column (GE Healthcare, US). The column was washed using washing buffer $(50 \mathrm{mM}$
$\mathrm{NaH}_{2} \mathrm{PO}_{4}, 300 \mathrm{mM} \mathrm{NaCl}, 20 \mathrm{mM}$ imidazole) to elute unbound proteins. The recombinant His-tagged rNS1 was eluted using elution buffer $\left(50 \mathrm{mM} \mathrm{NaH} \mathrm{PO}_{4}, 300\right.$ $\mathrm{mM} \mathrm{NaCl}$, and $250 \mathrm{mM}$ imidazole). The purification process was confirmed by $12 \%$ SDS-PAGE and western blot analysis. The purified rNS1 concentration was determined using a BCA Protein Assay Kit (Thermo, USA) in accordance to the manufacturer's protocol.

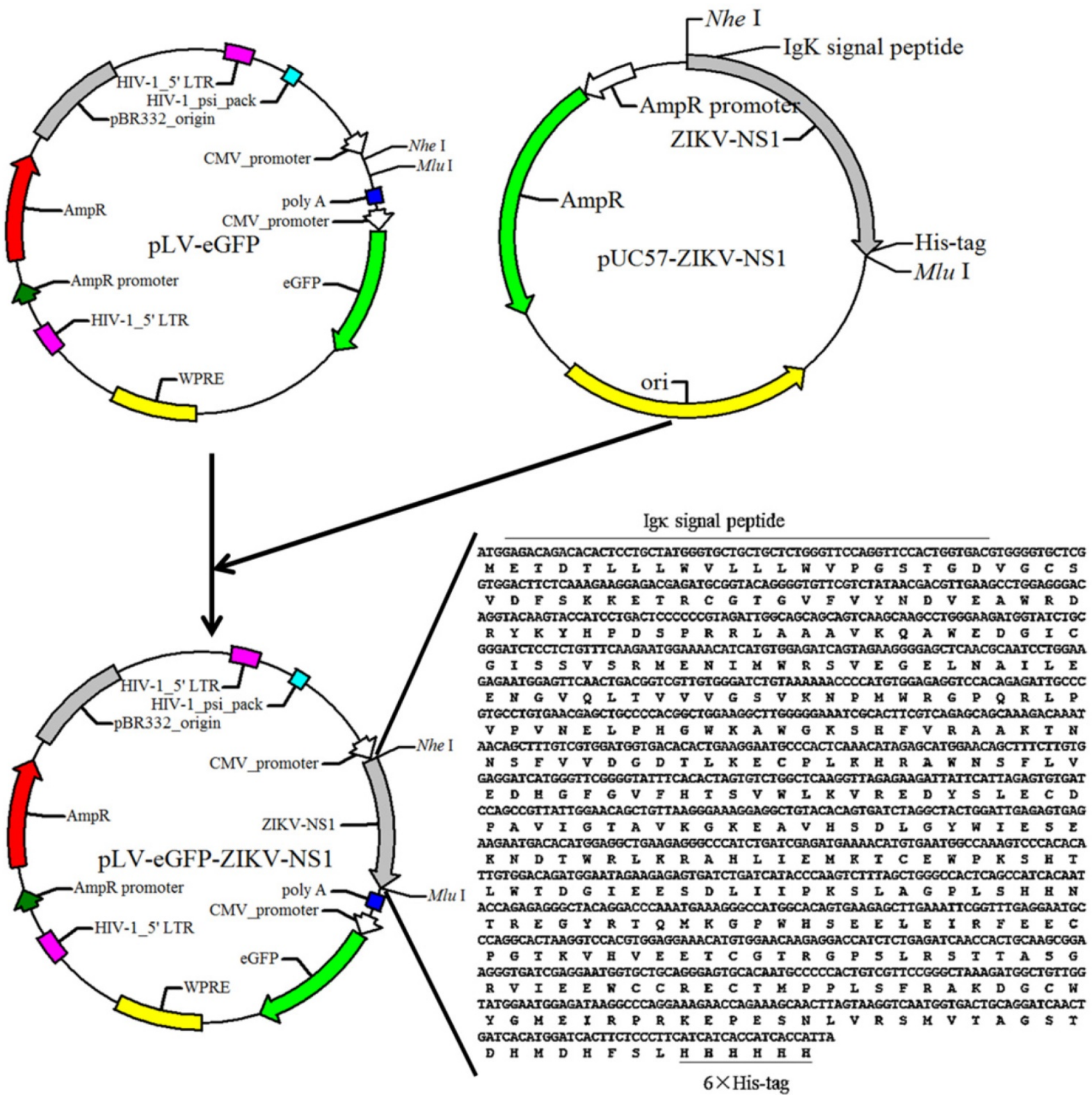

Figure 1. Diagram of the constructed recombinant plasmid pLV-eGFP-ZIKV-NS1. Sequence of synthesized cDNA was listed in the lower right corner, and the black lines indicate the lgK signal peptide and $6 \times$ his-tag. 


\section{Immunizations of mice and serum sample collection}

Mice immunization was performed in Laboratory Animal Center, Southern Medical University, Guangzhou, China. Ethical approval for the use of laboratory animal was obtained from Animal Care and Use Committee (ACUC) of Southern Medical University. 18 female BALB/c mice, aged 5 weeks, were randomly divided into three groups: low-dose group $(n=6$, subcutaneously injected with 5 $\mu \mathrm{g}$ rNS1), high-dose group $(\mathrm{n}=6$, subcutaneously injected with $20 \mu \mathrm{g}$ rNS1), and control group $(\mathrm{n}=6$, subcutaneously injected with equal volume of phosphate buffered solution (PBS)). After an interval of 2 weeks, mice were boosted with the same dose of prime vaccination. Seven days after prime and boost immunization, blood samples were obtained from the retroorbital plexus by a heparinized capillary tube, collected in an Eppendorf tube, and centrifuged, and serum was obtained and stored at $-20^{\circ} \mathrm{C}$.

\section{Measurement of antibody level}

ELISA was performed to evaluate level of ZIKV NS1-specific antibodies in blood samples of rNS1-immunzed mice. Briefly, $0.5 \mathrm{ng} / \mu \mathrm{l}$ purified rNS1, diluted with coating buffer was added in immunosorbent plates (Costar ${ }^{\circledR}$, Corning, US), incubated overnight at $4{ }^{\circ} \mathrm{C}$, and then washed four times with PBS (pH 7.4) containing $0.05 \%$ Tween 20 (PBST). To avoid non-specific binging, 5\% skim-milk was used to block plates. Plasma samples were diluted to 1:100 in PBS containing 5\% skim-milk, added in triplicate to microtiter plates (100 $\mu \mathrm{l} /$ well), and incubated at $37^{\circ} \mathrm{C}$ for $2 \mathrm{~h}$. The plates were then washed four times in PBST and incubated with horseradish peroxidase (HRP)-conjugated goat anti-mouse antibodies (Abcam, USA) diluted to 1:15,000 in PBST for $1 \mathrm{~h}$ at $37^{\circ} \mathrm{C}$. The plates were washed four times with a PBST washing solution. Tetramethylbenzidine substrate (TMB) solution (Thermo, USA) was added to each well and incubated for $15 \mathrm{~min}$. The reaction was stopped with $50 \mu \mathrm{l}$ of $1 \mathrm{M}$ sulfuric acid $\left(\mathrm{H}_{2} \mathrm{SO}_{4}\right)$ per well. The plates were read at $450 \mathrm{~nm}$ with microplate reader (Bio-Rad, USA).

\section{Western blot analysis}

Western blot were run as described previously [11]. Anti-NS1 and anti- $\beta$-actin monoclonal antibody were from Zoonogen and Cell Signaling Technology, respectively. Secondary antibodies horseradish peroxidase-conjugated anti-mouse IgG and anti-rabbit IgG were from Abcam.

\section{Statistical analysis}

All data were presented as the mean \pm standard deviation (SD). SPSS20.0 software was used for data analysis. The differences in mean values of the antibody levels between different groups were analyzed by one-way ANONA test. $p<0.05$ was considered to be statistically significant.

\section{Results}

\section{Construction of ZIKV NSI lentiviral expressing plasmid}

To express ZIKV NS1, a cDNA fragment encoding a chimeric protein, composed murine IgK signal peptide, NS1 and histidine tag was synthesized and then successfully subcloned into the lentiviral expression plasmid pLV-eGFP, resulting in the NS1 recombinant plasmid, pLV-eGFP-Zika-NS1 (Figure 1). The IgK signal peptide is designed to make the NS1 be secretory while the histidine tag is for purification of rNS1 using a nickel affinity column.

\section{Construction and identification of ZIKV NSI recombinant cell lines}

The 293T cells were transduced with LV-eGFP-Zika-NS1, and rNS1 and GFP expression was determined at $48 \mathrm{~h}$ post transduction (Figure 2A\&B). Total of 9 clonal cell lines of ZIKV NS1 were generated by limiting dilution with the seventh clone, HEK293T-ZIKV-NS1-7, showing the strongest secretory expression of rNS2 (Figure 2C\&D). The representative recombinant cell line maintained robust secretory NS1 expression for at least for 40 passages without significant difference in morphology, compared with untreated 293T cells. Western blot analysis using culture supernatants revealed that the HEK293T-ZIKV-NS1-7 cells stably expressed and secreted high levels of approximately 45-kDa NS1, and no significant changes were observed in GFP fluorescence ratio and fluorescence intensity (Figure 2E \&F)

\section{Purification of rNS1}

The recombinant NS1 protein tagged with the $6 \mathrm{x}$ histidine expressed in the supernatants of stable HEK293T-ZIKV-NS1-7 cells was purified using a nickel affinity column. Two distinct peaks were observed in the nickel affinity purification chromatogram (Figure 3A). The purified S1 protein was detectable at a high level by SDS-PAGE, and this was confirmed by immunoblotting with anti-ZIKV NS1 antibody (Figure 3B). The scanned gels were analyzed using Image-Pro Plus 6.0 software, and the result showed that the purity of the obtained rNS1 was $99.42 \%$. The protein concentration was 0.75 $\mathrm{mg} / \mathrm{ml}$, which was determined using a BCA Protein Assay Kit (Figure 3C). 

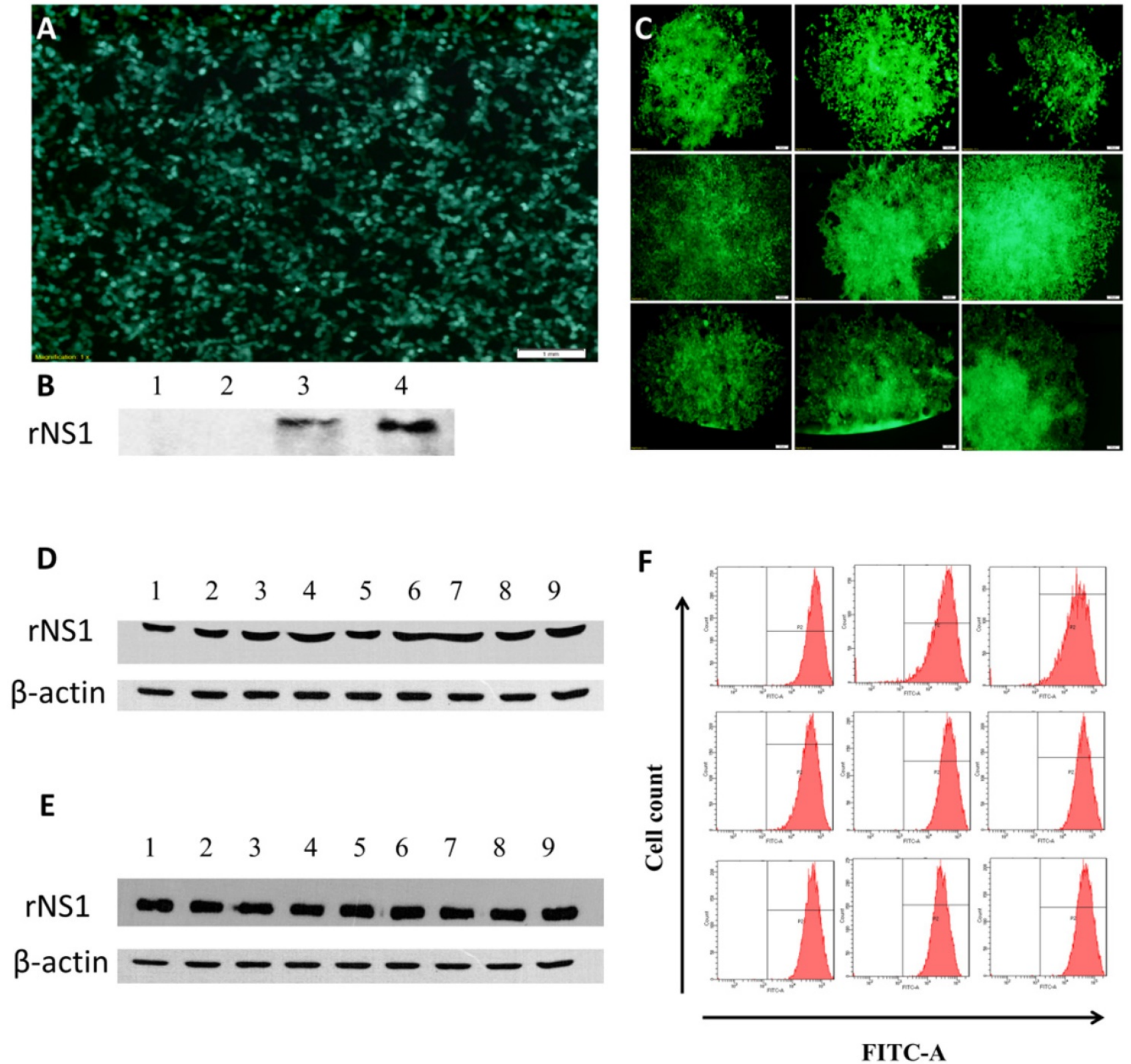

Figure 2. Construction and identification of recombinant cell lines. (A) Fluorescence microscopic images of GFP expression in HEK293T cells $48 \mathrm{~h}$ post-transduction. (B) Western blot results of $r$ NS1 expression $48 \mathrm{~h}$ post-transduction in HEK293T cells. Lane 1: Cell lysate of HEK293T cells transfected with pLV-eGFP; Lane 2: Supernatant of HEK293T cells transfected with pLV-eGFP; Lane 3: Cell lysate of HEK293T cells transfected with pLV-eGFP-ZIKV-NS1; Lane 4: Supernatant of HEK293T cells transfected with PLV-eGFP-ZIKV-NS1. (C) Fluorescence microscopic images of nine HEK293T cell lines that secrete rNS1. (D) Expression and secretion levels of rNS1 in 9 HEK293T monoclonal cells. Lanes 1 to 9: Cell culture supernatants of 9 HEK293T monoclonal cells. (E) Stability analysis of HEK293T-ZKKV-NS1-7 cells. Lanes 1 to 9: cell culture supernatants of HEK293T-ZKKV-NS1-7. Supernatants were collected every four generations. (F) Flow cytometry results of GFP fluorescence ratio and fluorescence intensity in HEK293T-ZIKV-NS1-7 cells. Flow cytometry analysis was carried out every four generation.

\section{Immunogenicity of ZIKV rNSI protein in mouse model}

Given the ability of commercial anti-ZIKV NS1 antibody to recognize the purified rNS1 protein, we then sought to determine whether the produced rNS1 can induce antibody production in mice. Mice were immunized as described in the Method section. Mouse antisera was collected at week 1 and 3 post-immunization and the presence of the anti-NS1 antibody in the sera was measured using ELISA. As seen in Figure 4, anti-ZIKV NS1 antibodies were barely detected in mice immunized with PBS. After the booster, significant increase concentrations of anti-NS1 antibodies were detected in immunized mice. Moreover, the mean levels of anti-NS1 antibodies in the high-dose group were significantly higher than in the low-dose group after the prime immunization. However, the anti-NS1 antibody levels of the two groups became similar after the booster immunization (Figure 4B). 


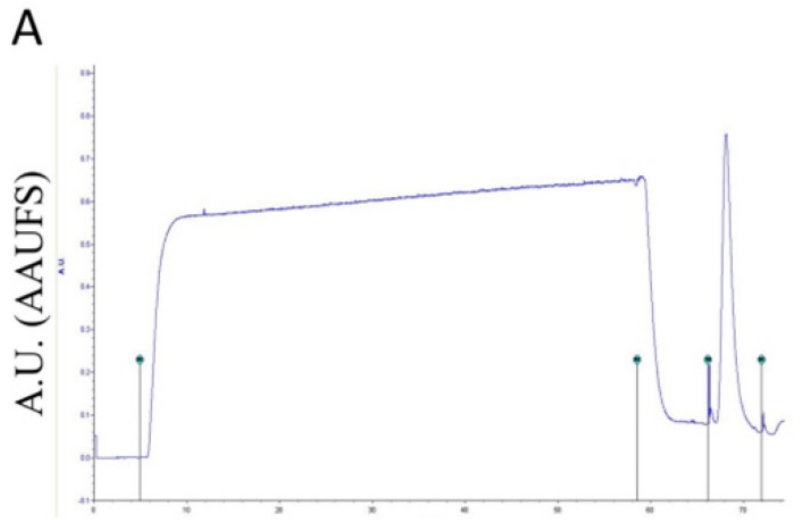

Time $(\min )$

C

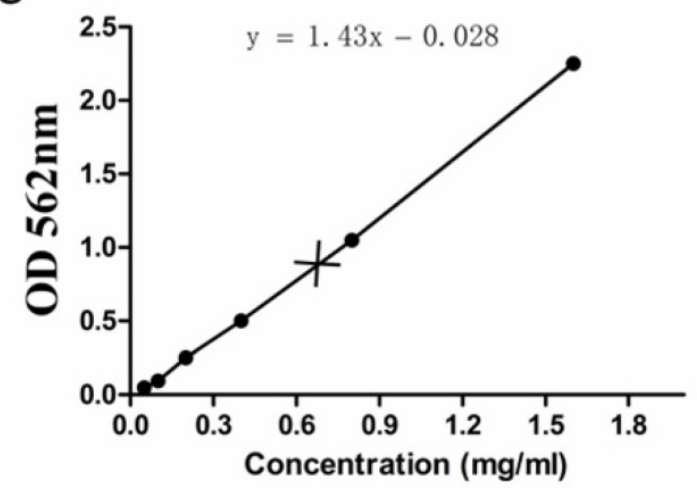

B

M \#1 \#2 \#3

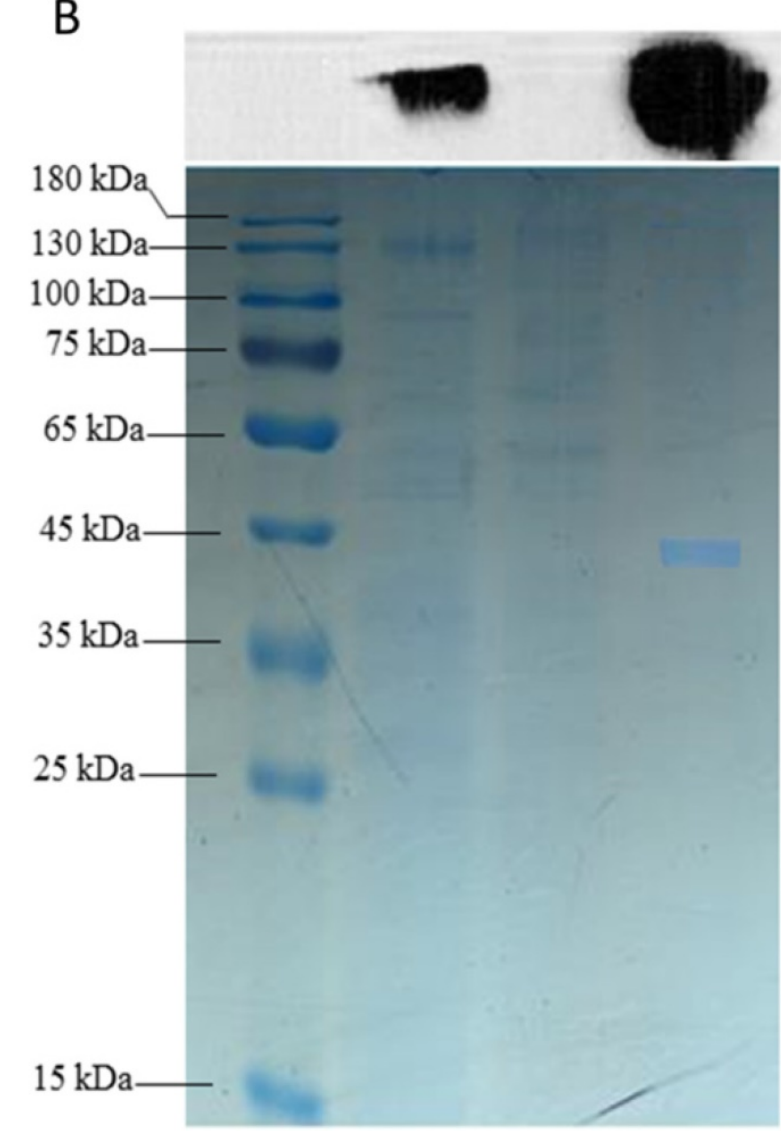

Figure 3. Purification of $r N S 1$. (A) Total protein expressed in HEK293T cells was purified by the BioLogic LP protein purification system using nickel affinity column. (B) Western blot and SDS-PAGE analysis of purification products. Lane M: protein marker; Lane \#1: total protein; Lane \#2: filtrate; Lane \#3: purification of expressed proteins using nickel affinity column. (C) Concentration of purified rNSI detected using BCA Protein Assay Kit.

A

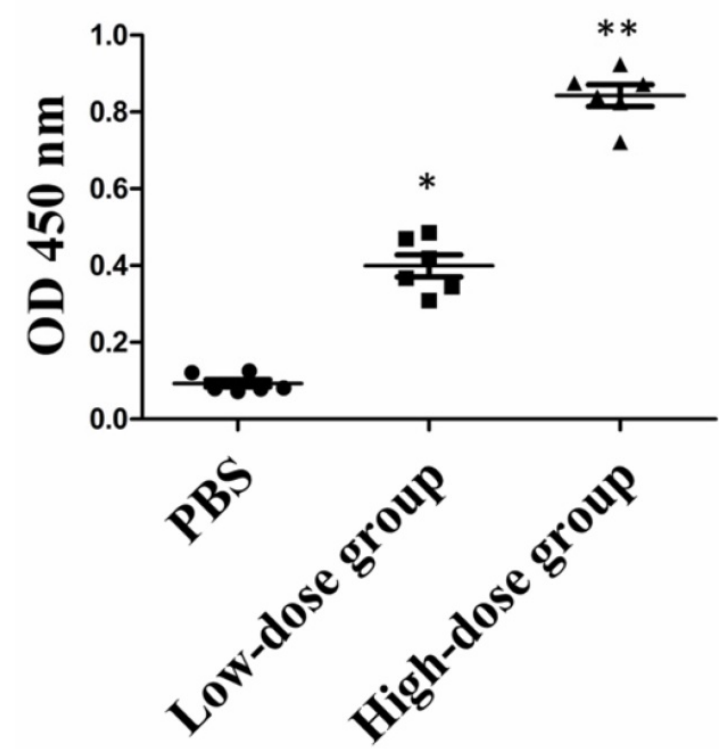

B

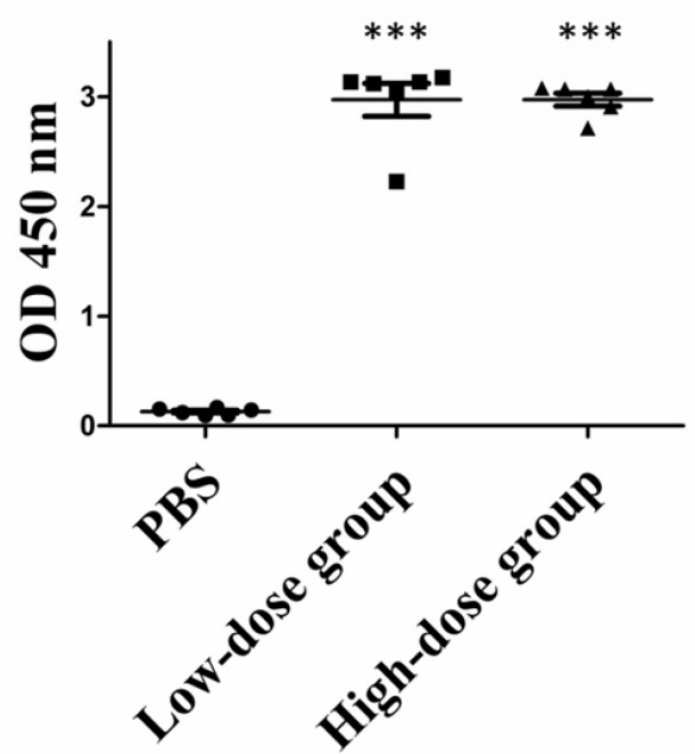

Figure 4. Serum antibody levels of $r N S 1$-immunzed mice. (A) Prime immunization. *: $\mathrm{P}<0.01$ versus with $\mathrm{PBS}$ group; **: $\mathrm{P}<0.01$ versus with low-dose group. (B) Booster immunization. ${ }^{*} * *: \mathrm{P}<0.01$ versus with $\mathrm{PBS}$ group. 


\section{Discussion}

ZIKV infection has become one of the most significant mosquito-borne infectious disease with significant burden to ZIKV-infected patients and society as a whole. In Latin American countries, health ministers made public recommendations to couples to postpone pregnancy from six months to two years in ZIKV epidemic areas [14-16]. With a broad range of clinical manifestations, early diagnosis of the disease remains crucial. Among the available dengue diagnosis tools, the detection of virus NS1 protein antigen has become the basis for commercial diagnostic assays [17]. However, there is no officially approved commercial kit for ZIKV serological diagnosis. We report here the production and purification of ZIKV rNS1 in mammalian cell expression system for the development of ZIKV serologic tests.

Previous studies have demonstrated that NS1 protein is essential for dengue virus replication and viability $[18,19]$. Many tests have been developed to diagnose dengue virus infections using NS1 due to the presence of the virus and their encoded NS1 protein in the blood during acute phase. Additionally, Oliveira et al. reported that DNA vaccine based the dengue-NS1 antigen induced $\mathrm{T}$ cell-mediated immunity, and protected mice during challenge by dengue virus [20]. Karin Stettler and colleagues reported that of 41 NS1 monoclonal antibodies, isolated from four ZIKV infected patients, only $22.0 \%$ monoclonal antibodies showed low cross-reactivity with dengue virus [21]. These results indicated that ZIKV NS1 has great potential for developing differential diagnosis test for ZIKV.

In last two decades, mammalian cell protein expression has become the dominant recombinant protein production system for clinical applications, producing more than half of the biopharmaceutical products in the market and several hundreds of candidates in clinical development. Expression systems for recombinant proteins using mammalian cells are able to introduce proper protein folding, post-translational modifications, which are often essential for full biological activity. In present study, nine of clones of HEK293T cells secreting rNS1 were generated based on a rNS1 lentivrial expressing vector using limiting dilution cloning. Stable expression of recombinant ZIKV NS1 was identified in the representative recombinant cell line, HEK293T-ZIKV-NS1-7, by western blotting assay using a commercial anti-NS1 antibody. The produced rNS1 proteins purified from the cell line were then used as antigens in mouse immunization. The recombinant proteins were injected subcutaneously.
Following the antigens administration, the collected serum from mice blood samples showed the presence of mouse anti-NS1 antibodies detected by ELISA method hence showed the immunogenicity of the recombinant NS1 proteins (Figure 4) and that the antibody generated from the immunization can recognize NS1 antigen.

In conclusion, we have generated and characterized recombinant ZIKV NS1 antigens, which were able to properly generate antibodies specific to ZIKV NS1 proteins and could be potentially used in the development of ZIKV NS1 diagnostic test. Moreover, the established ZIKV NS1 recombinant cell line, HEK293T-ZIKV-NS1-7, could be used as a cell model for studying biological characteristics and functions of NS1, a major host-interaction molecule that functions in flaviviral replication, pathogenesis, and immune evasion

\section{Acknowledgements}

We thank Dr. Junming Yue (Department of Pathology, University of Tennessee Health Science Center) for providing the vector pMD2.G and psPAX2.

\section{Funding}

This study was financially supported by the National Key R\&D Projects grant 2017YFD0500600, the Industrial High-tech Fields of Science and Technology Plan Projects of Guangdong Province grant 2013B010404026, the Science and Technology Planning Project of Guangdong Province, Grant 2010A011200003, 2012B011000004, 2016A030303008, 2012B060300003, the International S\&T Cooperation Program of China grant 2011DFA33290, the Medical Scientific Research Foundation of Guangdong Province, Grant A2017124.

\section{Authors' contributions}

Hongwei Li, Haifa Zheng and Weiwang Gu conceived and designed the study; Jun Liu, Andrew $\mathrm{Li}$ and Hongwei Li wrote the manuscript; Jun Liu, Pengfei Wang and Qingqing $\mathrm{Li}$ performed the majority of experiments; Jingjing $\mathrm{Li}$, Xiaoxin $\mathrm{Li}$, Huangyao Chen and Wenhan Liang contributed to the sample collection.

\section{Availability of data and materials}

The datasets used and/or analysed during the current study available from the corresponding author on reasonable request.

\section{Ethics approval and consent to participate}

Ethical approval for the use of laboratory animal was obtained from Animal Care and Use Committee 
(ACUC) of Southern Medical University. Ethical review number: N20160318.

\section{Competing Interests}

The authors have declared that no competing interest exists.

\section{References}

1. Oladapo OT, Souza JP, De Mucio B, De Leon RG, Perea W, Gulmezoglu AM. WHO interim guidance on pregnancy management in the context of Zika virus infection. Lancet Glob Health. 2016; 4:e510-1.

2. Franca GV, Schuler-Faccini L, Oliveira WK, Henriques CM, Carmo EH, Pedi VD, et al. Congenital Zika virus syndrome in Brazil: a case series of the first 1501 livebirths with complete investigation. Lancet. 2016; 388:891-7.

3. Vargas A, Saad E, Dimech GS, Santos RH, Sivini MA, Albuquerque LC, et al. Characteristics of the first cases of microcephaly possibly related to Zika virus reported in the Metropolitan Region of Recife, Pernambuco State, Brazil. Epidemiol Serv Saude. 2016; 25:691-700.

4. Machado-Alba JE, Machado-Duque ME, Gaviria-Mendoza A, Orozco-Giraldo V. Diagnosis of neurological disorders and the Zika virus epidemic in Colombia 2014 -2016. Int J Infect Dis. 2016; 51:133-4.

5. Parra B, Lizarazo J, Jimenez-Arango JA, Zea-Vera AF, Gonzalez-Manrique G, Vargas J, et al. Guillain-Barre Syndrome Associated with Zika Virus Infection in Colombia. N Engl J Med. 2016; 375:1513-23.

6. Pierson TC, Graham BS. Zika Virus: Immunity and Vaccine Development. Cell. 2016; 167:625-31.

7. Cecchetto J, Fernandes FC, Lopes R, Bueno PR. The capacitive sensing of NS1 Flavivirus biomarker. Biosens Bioelectron. 2017; 87:949-56.

8. Matheus S, Boukhari R, Labeau B, Ernault V, Bremand L, Kazanji M, et al. Specificity of Dengue NS1 Antigen in Differential Diagnosis of Dengue and Zika Virus Infection. Emerg Infect Dis. 2016; 22:1691-3.

9. Galula JU, Chang GJ, Chuang ST, Chao DY. Establishment of an Algorithm Using prM/E- and NS1-Specific IgM Antibody-Capture Enzyme-Linked Immunosorbent Assays in Diagnosis of Japanese Encephalitis Virus and West Nile Virus Infections in Humans. J Clin Microbiol. 2016; 54:412-22.

10. Song H, Qi J, Haywood J, Shi Y, Gao GF. Zika virus NS1 structure reveals diversity of electrostatic surfaces among flaviviruses. Nat Struct Mol Biol. 2016; 5:456-8.

11. Amorim JH, Porchia BF, Balan A, Cavalcante RC, Da CS, de Barcelos AA, et al. Refolded dengue virus type 2 NS1 protein expressed in Escherichia coli preserves structural and immunological properties of the native protein. J Virol Methods. 2010; 167:186-92.

12. He Y, Yan D, Zheng D, Hu Z, Li H, Li J. Cell Division Cycle 6 Promotes Mitotic Slippage and Contributes to Drug Resistance in Paclitaxel-Treated Cancer Cells. PLoS One. 2016; 11:e162-633.

13. Mao Y, Yan R, Li A, Zhang Y, Li J, Du H, et al. Lentiviral Vectors Mediate Long-Term and High Efficiency Transgene Expression in HEK 293T cells. Int J Med Sci. 2015; 5:407-15.

14. Burke A, Moreau C. Family Planning and Zika Virus: The Power of Prevention. Semin Reprod Med. 2016; 34:305-12.

15. Bahamondes L, Ali M, Monteiro I, Fernandes A. Contraceptive sales in the setting of the Zika virus epidemic. Hum Reprod. 2017; 32:88-93.

16. Tang WW, Young MP, Mamidi A, Regla-Nava JA, Kim K, Shresta S. A Mouse Model of Zika Virus Sexual Transmission and Vaginal Viral Replication. Cell Rep. 2016; 17:3091-98.

17. Simmons CP, Farrar JJ, Nguyen V, Wills B. Dengue. N Engl J Med. 2012; 366:1423-32.

18. Liu J, Liu Y, Nie K, Du S, Qiu J, Pang X, et al. Flavivirus NS1 protein in infected host sera enhances viral acquisition by mosquitoes. Nat Microbiol. 2016; 1:16087.

19. Conde JN, Da SE, Allonso D, Coelho DR, Andrade ID, de Medeiros LN, et al. Inhibition of the Membrane Attack Complex by Dengue Virus NS1 through Interaction with Vitronectin and Terminal Complement Proteins. J Virol. 2016; 90:9570-81.

20. Oliveira ER, Goncalves AJ, Costa SM, Azevedo AS, Mantuano-Barradas M, Nogueira AC, et al. Aspects of T Cell-Mediated Immunity Induced in Mice by a DNA Vaccine Based on the Dengue-NS1 Antigen after Challenge by the Intracerebral Route. PLoS One. 2016; 11:e163240.

21. Stettler K, Beltramello M, Espinosa DA, Graham V, Cassotta A, Bianchi S, et al. Specificity, cross-reactivity, and function of antibodies elicited by Zika virus infection. Science. 2016; 353:823-6. 